\title{
Non-symmetric gravity waves on water of infinite depth
}

\author{
By JUAN A. ZUFIRIA \\ Applied Mathematics Department, California Institute of Technology, Pasadena, \\ CA 91125, USA
}

(Received 13 August 1986)

Two different numerical methods are used to demonstrate the existence of and calculate non-symmetric gravity waves on deep water. It is found that they appear via spontaneous symmetry-breaking bifurcations from symmetric waves. The structure of the bifurcation tree is the same as the one found by Zufiria (1987) for waves on water of finite depth using a weakly nonlinear Hamiltonian model. One of the methods is based on the quadratic relations between the Stokes coefficients discovered by Longuet-Higgins (1978a). The other method is a new one based on the Hamiltonian structure of the water-wave problem.

\section{Introduction}

The problem of two-dimensional irrotational water waves of permanent form was first considered by Stokes (1849). He found, by using successive approximation methods, periodic travelling-wave solutions. Stokes's results were limited to smallamplitude waves. Another important contribution of Stokes in this field is his argument that if a sharp crest is attained by these waves as the amplitude is increased, the sharp crest must have an angle of $120^{\circ}$. During the last decade, with the use of computers, Stokes's solutions have been extended to finite-amplitude waves. It has also been proved that Stokes's argument concerning the $120^{\circ}$ singular crest is true (Amick, Fraenkel \& Toland 1982).

For the case of finite depth, where shallow-water approximations have made it possible to introduce simplified models accessible to analytical tools, not only have periodic families been found for finite depth (cnoidal waves), but also solitary-wave solutions have been found (Boussinesq 1871; Rayleigh 1876; Korteweg \& de Vries 1895).

If $x$ is taken to be the horizontal coordinate, the equations for travelling waves are invariant under the change $x \rightarrow-x$. This relation defines a symmetry group. A large class of solutions of the equations are invariant under the action of the same symmetry group. These solutions are called symmetric. In other words, a wave is said to be symmetric when, if $f(x)$ represents the shape of the wave, the origin of the $x$-axis can be chosen such that $f(x)=f(-x)$. All the travelling-wave solutions that are known to date correspond to symmetric waves.

It is very well known in physics that bifurcations can lead to solutions that are no longer invariant under the action of the symmetry group. Two typical examples are the Hopf bifurcation and Bénard convection. In a Hopf bifurcation the temporal symmetry is broken, and in Bénard convection the spatial symmetry is broken with the appearance of hexagonal convection cells. Bifurcation in the presence of symmetries has been extensively studied by different authors using grouprepresentation theory (Sattinger 1980, 1983). 
The possibility of finding non-symmetric solutions for the problem of water waves has been until now an open question. Another open question is, given that these solutions exist, do they appear via a spontaneous symmetry-breaking bifurcation from a symmetric family or as isolated branches of solutions?

The rapidly increasing power of numerical methods to study finite-amplitude waves has encouraged some authors to search for these kinds of solutions in such waves. Chen \& Saffman (1980) studied finite-amplitude gravity waves on deep water by numerical methods. They found that gravity waves of finite amplitude are not unique. Bifurcations to families of waves in which all crests are not equal were found. They computed two new families which they called irregular class 2 and class 3 waves. These waves are still symmetric. They did find symmetry-breaking bifurcations of the equations, but the new solutions proved to be just shifted symmetric waves. In later work, Saffman (1980), using a method based on the quadratic relations between the Stokes coefficients discovered by Longuet-Higgins (1978a), demonstrated that an infinite set of bifurcations to new families exists for the Stokes family, but all the new branches correspond to symmetric waves. Also Longuet-Higgins (1985), using the same method, showed that there are no bifurcations into non-symmetric waves from the regular Stokes family, over a certain range.

Recently Zufiria (1987), using a weakly nonlinear Hamiltonian model based on small-amplitude and dispersion approximations, found that for water of finite depth families of non-symmetric waves are possible. These waves appear via spontaneous symmetry-breaking bifurcations from families of symmetric waves. His results follow from some universal behaviour found by Green etal. (1981) on the bifurcation properties of fixed points of two-dimensional area-preserving maps in the presence of one symmetry. In particular Green et al. (1981) were able to locate some symmetrybreaking bifurcations. Zufiria's (1987) Hamiltonian model could be reduced to a two-degrees-of-freedom discrete Hamiltonian system, and thus via surface section can be reduced to an area-preserving map (Arnol'd \& Avez 1968).

Zufiria's (1987) results raise several questions. The first is whether these results can be generalized to the full equations or are just properties of the approximation. Another question is whether these results hold for deep-water waves, where assumptions of small amplitude-depth ratio and low dispersion are no longer valid.

In this paper, motivated by these questions, we compute finite-amplitude waves on deep water. We show that the same bifurcation scenario occurs. Therefore non-symmetric permanent-form travelling waves on deep water exist, and they appear via symmetry-breaking bifurcations. To confirm our results we have done the computations using two different methods, and we have found the same results.

The first method that we consider is the one used by Longuet-Higgins (1985) and Saffman (1980). It consists of an expansion of the shape of the wave in the potential plane, and is based on Longuet-Higgins's (1978a) quadratic relation. This is a very simple method, gives the most accurate results, and can be very easily implemented.

The second method is based in the Hamiltonian structure of the water-waves problem. Because the results found by Zufiria (1987) on finite depth are related to the Hamiltonian structure of the problem, we thought that it would be interesting to develop a numerical method that kept the Hamiltonian structure of the original formulation. That method would contain all the symmetries of the problem, and the bifurcation scenario would perhaps be better obtained.

The Hamiltonian method of computation is more complicated and time-consuming, but it offers some other advantages. The first is that the superharmonic stability of 
the wave solutions can be computed directly without the need to write a more complicated code, as happens in other formulations. This is true because the Jacobian of the set of equations is the stability matrix, as we shall see below. A second advantage is that a small number of modes suffice to reproduce the bifurcation behaviour of the problem, whereas in the Stokes expansion method it is found (P. G. Saffman, private communication) that a minimum number of around 50 modes in a class 1 wave are needed to reproduce the bifurcation structure of the problem.

Another motivation to try this numerical approach came from the successful results obtained by several authors during the last few years on the stability of travelling waves using the Hamiltonian formulation for the water-waves problem. Some examples are Saffman (1985), MacKay \& Saffman (1986), Zufiria \& Saffman (1986).

\section{Stokes expansion}

\subsection{General formulation}

Consider two-dimensional irrotational surfaces waves of permanent form travelling with speed $c$ in the negative $x$-direction on water of infinite depth. In a frame of reference moving with the wave, following Stokes (1849) and taking the basic wavelength to be $2 \pi$, the $x$-and $y$-coordinates of the flow can be expressed in the following form.(Longuet-Higgins 1985):

$$
x+\mathrm{i} y=\frac{1}{c}(\Phi+\mathrm{i} \Psi)+\frac{1}{2} \mathrm{i} a_{0}+\mathrm{i} \sum_{k=1}^{\infty} a_{k} \mathrm{e}^{\mathrm{i} k(\Phi+\mathrm{i} \Psi) / c},
$$

where $\Phi$ and $\Psi$ are the velocity potential and the stream function respectively.

For a general wave the coefficients $a_{k}$ are complex, except for $a_{0}$, which can always be taken to be real. In the case of symmetric waves the origin of the frame of reference can always be chosen such that all the coefficients $a_{k}$ are real. This happens when the origin of the reference frame lies on one of the axes of symmetry of the wavetrain.

If the horizontal level of the frame of reference is chosen such that the mean surface level is $\bar{y}=-c^{2} / 2 g$, Longuet-Higgins $(1978 a, 1985)$ has shown that the dynamical boundary condition of constant pressure at the free surface $(\Psi=0)$ is equivalent to the set of equations

$$
\int_{\Phi=0}^{2 \pi c} y \mathrm{e}^{-\mathrm{i} k \Phi / c}(\mathrm{~d} x+\mathrm{i} \mathrm{d} y)=\left\{\begin{array}{cl}
-\pi c^{2} & (k=0) \\
0 & (k=1,2, \ldots)
\end{array}\right.
$$

where $g=1$ has been taken.

Introducing (1) into (2) we obtain the following set of quadratic equations for the coefficients :

$$
\begin{gathered}
a_{0}+\sum_{k=1}^{\infty} k a_{k} a_{k}^{*}=-c^{2} \quad(m=0), \\
\left(1+m a_{0}\right) a_{m}+\sum_{k=1}^{m-1} k a_{k} a_{m-k}+\sum_{k=1}^{\infty}(k+m) a_{k+m} a_{k}^{*}=0 \quad(m>0),
\end{gathered}
$$

where the asterisk denotes complex conjugate. The first equation is real and the rest are complex. For computational purposes and for the following discussion it is 
convenient to split the complex equations into real and imaginary parts. Taking $a_{k}=p_{k}+\mathrm{i} q_{k}$, we have

$$
\begin{aligned}
& p_{m}\left(1+m a_{0}\right)+\sum_{k=1}^{m-1} k\left(p_{k} p_{m-k}-q_{k} q_{m-k}\right)+\sum_{k=1}^{\infty}(k+m)\left(p_{k+m} p_{k}+q_{k} q_{k+m}\right)=0 \\
& q_{m}\left(1+m a_{0}\right)+\sum_{k=1}^{m-1} k\left(p_{k} q_{m-k}+q_{k} p_{m-k}\right)-\sum_{k=1}^{\infty}(k+m)\left(p_{k+m} q_{k}-p_{k} q_{k+m}\right)=0
\end{aligned}
$$

If we truncate the expansion (1) at the term $a_{n}$ and take $a_{k}=0$ for $k>n,(3 a)$ together with the set of equations (4) form a system of $2 n+1$ nonlinear algebraic equations with $2 n+1$ unknowns $\left(a_{0}, p_{k}, q_{k}\right), c$ being the only parameter of the problem.

This scheme, simplified for symmetric waves, has been used by several authors. For the family of Stokes waves that we shall call regular waves because all the crests and troughs are equal, the phase speed $c$ does not behave monotonically through the full range of steepness. This has led several authors to introduce other parameters that behave monotonically for the full range of steepness. Longuet-Higgins (1985) used the parameter $Q_{\mathbf{c}}$ defined as follows

$$
Q_{\mathrm{c}}=1-\frac{1}{2} v_{\text {crest }}^{2},
$$

where $v_{\text {crest }}$ is the velocity at the crest of the wave. This parameter ranges from $\frac{1}{2}$ to $1: Q_{\mathrm{c}}=\frac{1}{2}$ corresponds to the flat surface, and $Q_{\mathrm{c}}=1$ to the $120^{\circ}$ Stokes limiting wave.

Chen \& Saffman (1980) used another parameter that they called $b$. This parameter $b$ is defined and related to $Q_{c}$ through

$$
b=1-\frac{v_{\mathrm{crest}}^{2}}{c^{2}}=1-\frac{2\left(1-Q_{\mathrm{c}}\right)}{c^{2}} .
$$

We introduce the parameter $Q$ as

$$
Q=1+y_{0}=1+\frac{1}{2} a_{0}+\sum_{k=1}^{n} p_{k},
$$

where $y_{0}$ is the height of the wave at the origin of the $x$-axis. When the origin is chosen on the crest, $Q=Q_{\mathrm{c}}$.

Equation (7) can be used to complete system (4) instead of using the equation for $c$, and then the Stokes family can be studied taking $Q$ as parameter.

As we shall see below, some of the bifurcations that we find break the symmetry with respect to the crests, but not with respect to the troughs. Therefore it is convenient to take the origin of $x$ on a trough. In our computations we took the origin on a trough and used (7) to complete the system.

A property of $(3)$ is that if a set of coefficients $\left\{a_{k}\right\}$ represents a wave, the set $\left\{\hat{a}_{k}^{N}\right\}$ where

$$
\left.\begin{array}{rl}
\hat{a}_{0}^{N} & =\frac{a_{0}}{N}, \\
\hat{a}_{N k}^{N} & =\frac{a_{k}}{N}, \\
\hat{a}_{k}^{N} & =0 \quad \text { otherwise, }
\end{array}\right\}
$$

is also a solution that describes the same wave but of a different scale. The set $\left\{a_{k}\right\}$ represents a wave of wavelength $2 \pi$, and the set $\left\{\hat{a}_{k}^{N}\right\}$ represents the same wave with 
wavelength $2 \pi / N$. These waves are called class $N$ waves (Saffman 1980) because there are $N$ crests in the window of $x$ between 0 and $2 \pi$.

Another property of the system defined by (3) is that it is degenerate in the sense that solutions are not isolated. Due to the Galilean invariance, if a set of coefficients $\left\{a_{k}\right\}$ satisfies (3) the set $\left\{a_{k} \mathrm{e}^{\mathrm{i} k \epsilon}\right\}$ is also a solution of (3) for all $\epsilon$. So for a given value of $Q,(3)$ do not uniquely determine a solution. To remove this degeneracy we thus need to fix the phase. As we shall see below, this degeneracy disappears when considering symmetric waves. To avoid this problem for non-symmetric waves we shall introduce some modifications into the system of equations.

Because Zufiria (1987) found the symmetry breaking from a class 6 wave, we shall start computing with a regular wave of class 6 . Therefore the coefficient $a_{6}$ will be the dominant coefficient in a solution set $\left\{a_{k}\right\}$. To avoid the Galilean-invariance degeneracy, we shall force $a_{6}$ to be real $\left(q_{6}=0\right)$ in our computations. This was actually implemented by modifying the equation corresponding to $a_{6}$ in (3) to the following form:

$$
q_{6}+a_{6}\left(1+6 a_{0}\right)+\sum_{k=1}^{5} k a_{k} a_{6-k}+\sum_{k=1}^{\infty}(6+k) a_{6+k} a_{k}^{*}=0 .
$$

The modified system has the same solutions as the original system if $q_{6}=0$, and the new system is not degenerate any more. This simple modification has been found to work very well during our computations.

As we are to study bifurcations, it is important to examine the Jacobian of the system defined by (4), which has the following form:

$$
\mathcal{J}(p, q)=\left(\begin{array}{ll}
A_{1}(p) & B(q) \\
B(q)^{\mathrm{T}} & A_{2}(p)
\end{array}\right),
$$

where $\boldsymbol{p}=\left\{p_{k}\right\}$ and $\boldsymbol{q}=\left\{q_{k}\right\}$. This Jacobian is always singular, and the eigenvector associated to the zero eigenvalue is the shift. $A s A_{1}$ and $A_{2}$ are symmetric $J$ is symmetric when $q=0$. This symmetry will simplify some of the computations.

Some properties of the waves, such as the kinetic and potential energies, can be expressed in terms of the set of coefficients $\left\{a_{k}\right\}$. For example, the kinetic energy per one wavelength in the frame of reference in which the fluid is at rest at the bottom $(y \rightarrow-\infty)$ is

where $(3 a)$ has been used.

$$
T=\frac{1}{2} \pi c^{2} \sum_{k=1}^{n} k a_{k} a_{k}^{*}=-\frac{1}{2} \pi c^{2}\left(a_{0}+c^{2}\right),
$$

The potential energy, taking the mean horizontal level of the surface as reference level, can be written as

$$
\begin{aligned}
V=\pi\{ & -\frac{1}{2}\left(c^{2}+a_{0}\right)^{2}+\frac{1}{2} \sum_{k=1}^{n} a_{k} a_{k}^{*} \\
& \left.+\frac{1}{8} \sum_{k=1}^{n} k\left[2 \sum_{j=1}^{n-k}\left(a_{k} a_{j} a_{k+j}^{*}+a_{k}^{*} a_{j}^{*} a k_{+j}\right)+\sum_{j=1}^{k-1}\left(a_{k} a_{j}^{*} a_{k-j}^{*}+a_{k}^{*} a_{j} a_{k-j}\right)\right]\right\} .
\end{aligned}
$$

A similar expression for $V$ was obtained by Longuet-Higgins (1984) for the case of symmetric waves.

\subsection{Symmetric waves}

All actual calculations that give waves have assumed symmetric waves owing to the inability to find non-symmetric solutions. When symmetric waves are considered, large simplifications can be introduced in the formulation of the problem. The first 
one is that the shift can be chosen such that $q_{k}=0$ for all $k$, and the truncated system of equations defined by (4) and (7) reduces to

$$
\left.\begin{array}{c}
p_{m}\left(1+m a_{0}\right)+\sum_{k=1}^{m-1} k p_{k} p_{m-k}+\sum_{k=1}^{n}(m+k) p_{m+k} p_{k}=0 \quad(m>0), \\
\frac{1}{2} a_{0}+\sum_{k=1}^{n} p_{k}=Q-1
\end{array}\right\}
$$

The degeneracy related to the Galilean invariance disappears because the shift is fixed by the condition $q=0$. As we have already pointed out in the general formulation, a wave appears as a continuous set of solutions by varying the shift $\epsilon$, but when the wave is symmetric only a discrete and isolated set of those solutions satisfies the condition $q=0$. For example, for a regular wave, if we take the origin of the $x$-axis at a trough or a crest we find $q=0$. The starting point for the calculations determines where the origin is taken.

If $q=0$, the Jacobian defined by relation $(10)$ is

$$
\mathcal{J}(p, 0)=\left(\begin{array}{cc}
\boldsymbol{A}_{1}(p) & 0 \\
0 & \boldsymbol{A}_{2}(p)
\end{array}\right)
$$

because $B(0)=0$. Notice that $\boldsymbol{A}_{1}(p)$ is the Jacobian that appears in the symmetric formulation given by (13). As Galilean invariance is not allowed in the symmetric formulation $\boldsymbol{A}_{1}(p)$ will not be singular in general. As $J(p, q)$ is always singular $\boldsymbol{A}_{2}(p)$ has to be always singular, and the null eigenvector will be the shift. If we now consider the modification introduced by $(9), \boldsymbol{A}_{1}(\boldsymbol{p})$ does not change, but $\boldsymbol{A}_{2}(\boldsymbol{p})$ does. Let us call the new matrix $A_{2}^{\prime}(p)$ and the new Jacobian $J^{\prime}(p, 0)$. These matrices will not be singular in general.

A bifurcation from a family of symmetric waves to a different family corresponds to a zero eigenvalue of $\boldsymbol{J}^{\prime}$. This zero eigenvalue can appear in two ways. One is via a zero eigenvalue of $\boldsymbol{A}_{1}$ that corresponds to a new family of waves that are symmetric again with respect to $x=0$. The second way is a zero eigenvalue of the matrix $\boldsymbol{A}_{2}^{\prime}$. This point corresponds to a bifurcation to a family of waves that are no longer symmetric with respect to $x=0$. This does not mean that the new branch corresponds necessarily to non-symmetric waves. As Chen \& Saffman found, these branches can correspond to symmetric waves for which the origin of $x$ does not lie on one of the axes of symmetry of the wave. But if non-symmetric waves exist and they appear through symmetry-breaking bifurcations from symmetric waves, the symmetrybreaking bifurcation has to correspond to a zero eigenvalue of $\boldsymbol{A}_{2}^{\prime}$.

For a given set of coefficients $\left\{a_{k}\right\}$, whether the set represents a symmetric or non-symmetric wave can be determined by computing the ratios $a_{k} / a_{k+1}$. If the wave is symmetric we find that $\arg \left(a_{k} / a_{k+1}\right)=$ const. for all $k$.

\subsection{Numerical results}

We started by computing the family of regular Stokes waves. Following Zufiria (1987), because of the type of structure that we expect in the bifurcation diagram, we start with a regular class 6 wave (six equal crests in the interval $(0,2 \pi)$ ). The starting point was generated using Schwartz's (1974) expansion for low-amplitude regular waves.

This branch is symmetric, and therefore the system defined by (13) was used in the computations. The origin of the $x$-axis was chosen in a trough. A simple arc-length (Keller 1977) continuation method was used, with $Q$ as the parameter along the branches. The algorithm converged very rapidly in all regions, reaching an error of 


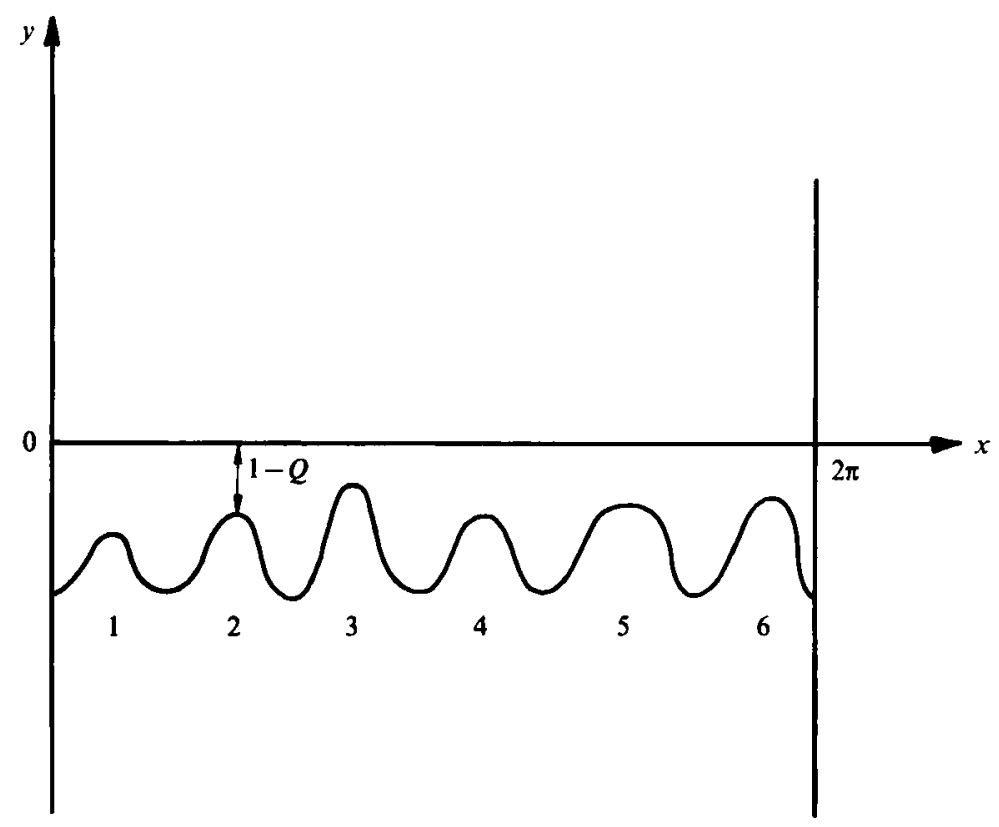

Figure 1. Periodic travelling wave of class 6.

less than $10^{-12}$ for the quadratic norm of the residues of the equations in three or four iterations for the step used.

Because we shall deal in our computations with families of waves for which the six crests are not equal, it is convenient to introduce a parameter to measure the amplitude of each of the six crests. As a generalization of (7) we define

$$
Q_{j}=1+y_{j}, \quad j=1,2,3,4,5,6,
$$

where $y_{j}$ is the height of the different crests (figure 1). Notice that if $Q_{j}=1$ for some $j$, this means that the $j$ th crest reaches the limiting $120^{\circ}$ Stokes crest. This is because $y=0$ corresponds to zero velocity from Bernoulli's equation.

To make the difference among the families of waves that we are going to study clearer, we introduce the following notation. We associate with each of the six crests one of the following letters $Z, Y, X, W, V, U$ depending on the relative amplitude of the crests, where $Z>Y>X>W>V>U$. A regular wave of class 6 will be denoted by six equal letters.

Computations have been performed using $n=256,512,768$ and in some cases up to 1024. We have found that for most of the branches in which we are interested $n=512$ gives good convergence results.

Because the bifurcations that we found are not simple, bifurcation points were detected during the computations by monitoring the determinant and the last three pivots of the Gaussian elimination for the matrices $\boldsymbol{A}_{1}$ and $\boldsymbol{A}_{2}^{\prime}$. In some cases, to understand the bifurcations better we also computed the eigenvalues and eigenvectors of $\boldsymbol{A}_{\mathbf{2}}$.

To switch branches a code based on Keller's (1977) method was written. But in most of the cases it was found that to switch branches it was enough to perturb the solution at the bifurcation point with the null vector, and impose the condition that the first few Newton iterations of the continuation process be normal to the null 


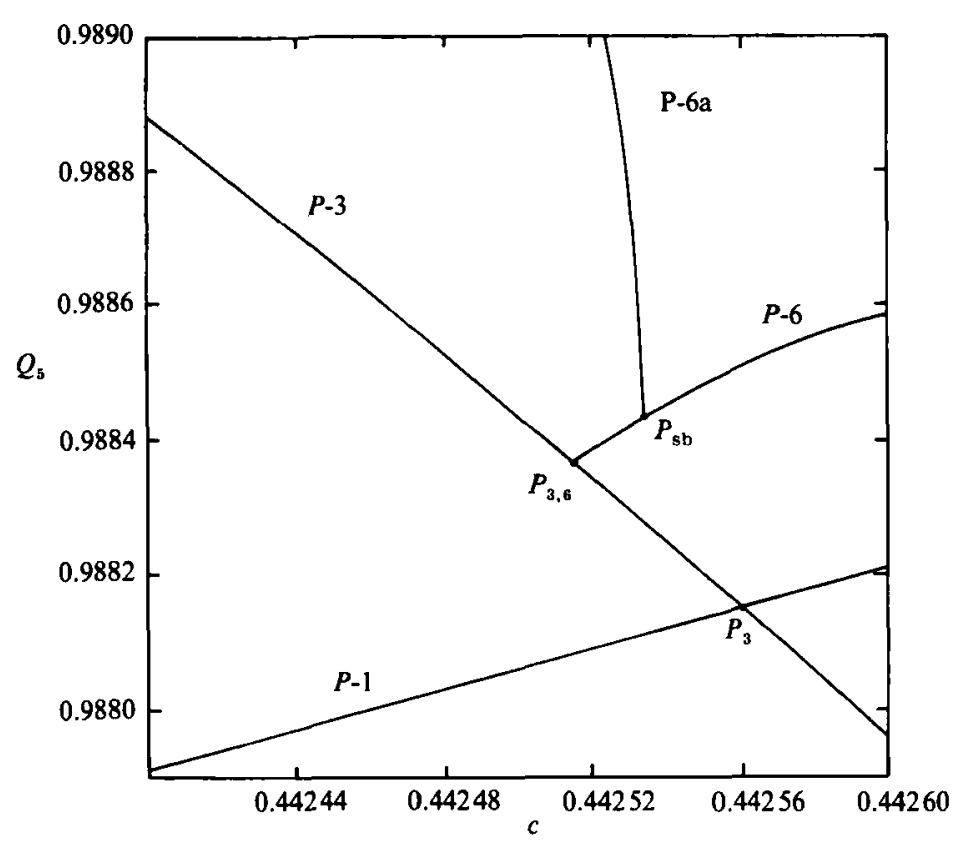

FIGURE 2. Bifurcation diagram for solution branches showing wave height versus wave speed for travelling waves of permanent form on deep water.

vector. This method was found to converge to the new branch in three or four iterations.

Longuet-Higgins (1985) showed that period-doubling and period-tripling bifurcations from the regular family appear in very truncated models $(n=2$ and 3 ) of (13). We have found that this is true, but these bifurcations disappear when the number of modes reaches 8 , and they do not appear again until the number of modes is around 200 for a class 6 wave.

Starting at low amplitude we computed the regular branch, that we will call the P-1 branch. For this branch the period of the wave is $L$, where $L=\frac{2}{6} \pi$. As the amplitude was increased the determinant of $\boldsymbol{A}_{1}$ was found to vanish at three points, these points corresponding to period-six, period-three and period-two bifurcations. We will refer to these points as $P_{6}, P_{3}$ and $P_{2}$ respectively. The point $P_{3}$ is shown in figure 2. These bifurcations correspond to the ones that Chen \& Saffman (1980) and Saffman (1980) found. $A_{2}^{\prime}$ was singular at the two points $P_{6}$ and $P_{3}$. But no non-symmetric families are created at these points. These branches just represent shifted symmetric waves.

The new family of waves that is created at the period-tripling bifurcation has the form $Y Z Y Y Z Y$ (see figure 3) and has wavelength $\pi$. The branches that appear as non-symmetric waves with respect to $x=0$ have the form $Z Y Y Z Y Y$ and $Y Y Z Y Y Z$, but are the same waves as $Y Z Y Y Z Y$. This $P-1$ branch was computed using $n=256$, 512 and 768 . Values of some parameters of the wave at the bifurcation point $P_{3}$ are given in table 1 . We can see that the agreement with the results of other authors and methods is good. The values obtained for the period-six and period-doubling bifurcations agree with those given by Saffman (1980) to four figures. In this table, $a k$ denotes the steepness, which is defined as one-half of the waveheight times the wavenumber for the regular class 1 wave.

With $n=512$, computations were carried out up to steeper waves without being 
(a)

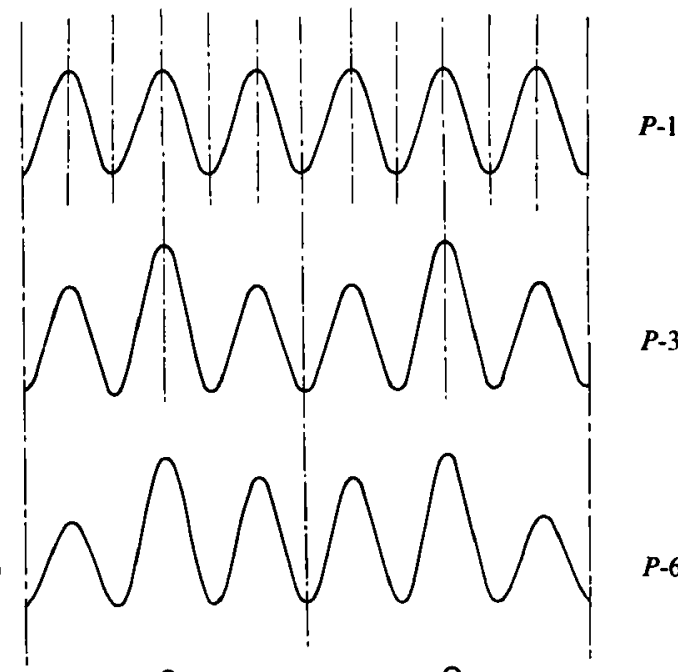

(d)

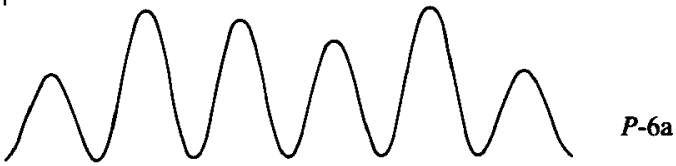

Figure 3. Schematic representation showing the relative amplitude of the crests of the travelling waves corresponding to different branches of the bifurcation diagram given in figure 2 .

Saffman (1980)

Chen \& Saffman (1980)

Longuet-Higgins (1984)

Simmen \& Saffman (1985)

Present work

Stokes expansion

$\begin{array}{ccccc}n & Q & c & E=T+V & a k \\ 300 \dagger & - & 0.4425 & 0.01239 & 0.40464 \\ - & 0.98815 & 0.44255 & 0.01239 & 0.40470 \\ 320 \dagger & - & - & - & 0.404688 \\ - & - & 0.4426 & - & 0.40470\end{array}$

$\begin{array}{rccll}256 & 0.98848 & 0.44306 & 0.012518 & 0.406644 \\ 512 & 0.98815 & 0.44261 & 0.012394 & 0.404676 \\ 768 & 0.98815 & 0.44255 & 0.012393 & 0.404676 \\ 48 & - & 0.44350 & 0.01266 & 0.40794 \\ 60 & - & 0.44268 & 0.01243 & 0.40440 \\ 72 & - & 0.44262 & 0.01240 & 0.404664\end{array}$

Table 1. Values of some parameters of the wave at the bifurcation point $\boldsymbol{P}_{3}$ + Modes on a class 1 wave.

able to find the first maximum in the energy. To obtain convergence in the strongly nonlinear region, it is necessary to increase the number of modes to around 300 for a class 1 wave (Longuet-Higgins 1985), which is equivalent to 1800 modes for a class 6 wave.

At the period-tripling bifurcation point $\left(P_{3}\right)$, we switched from the P-1 branch to the P-3 branch (period $3 L$ ). Taking the P-3 branch in the direction of decreasing $c$ from $P_{3}$, we found a point for which $A_{1}$ becomes singular, point $P_{3,6}$ in figure 2 . The bifurcation here corresponds to a period-doubling bifurcation. The new branch corresponds to a family of waves of the form $X Z Y Y Z X$. Notice now the importance 


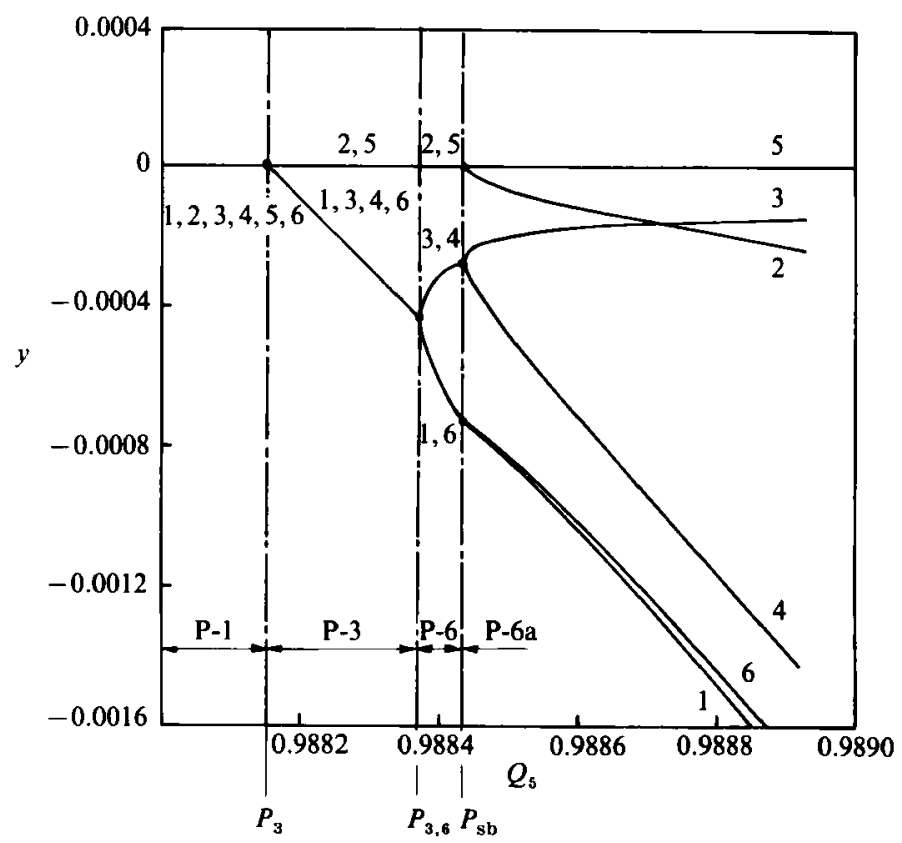

Fiaure 4. Relative amplitudes of the crests of the travelling waves along the path P-1, P-3, P-6 and P-6a. Numbers denote the crests of figure 1.

of taking the origin of the $x$-axis in a trough. If we had taken the origin in a crest, these waves would have appeared as a spurious non-symmetric branch. This is because the period-doubling bifurcation breaks the symmetry with respect to the crests, but not with respect to the troughs. With the origin in a crest, instead of finding $A_{1}$ to be singular, we would have found $A_{2}^{\prime}$ to be singular at $P_{3,6}$. Thus the new branch could not be computed using system (13). The new family of waves is not symmetric with respect to any crest, in contrast to the $P-1$ and P-3 branches where the waves were symmetric with respect to some crest (see figure 3 ).

On this new branch of period $6 L$, which we shall call the P-6 branch, computations were carried out in both directions from the bifurcation point. We found that the two sides correspond to the same wave, the difference being a shift of $\pi$ in the origin (see figure 3, curves $b$ and $c$ ). The matrix $A_{1}$ was non-singular on the whole computed region of the branch, except at the point $P_{3,6}$. It was also found that $\boldsymbol{A}_{2}^{\prime}$ vanishes at one point of this branch (point $P_{\mathrm{sb}}$ in figure 2). This means that at this point there is bifurcation to a family of waves that are not symmetric with respect to the trough at which we took the origin of $x$.

The new branch was examined with a non-symmetric code based on the system formed by (4) and (7). This time we found that the waves were not just shifted symmetric waves, as we found in the other cases in which $\boldsymbol{A}_{2}^{\prime}$ vanished. This new branch corresponds to a family of non-symmetric waves. At the beginning of the branch the waves have the form described by the sequence $W Z Y X Z W$ (see figure 3), but if we go further along the branch we find that all the crests have different heights. The difference among the heights of the crests is very small and cannot easily be seen by eye. Figure 4 shows the relative height of all the crests with respect to the fifth crest for all the branches that we have computed. We in fact plot $\left(Q_{j}-Q_{5}\right)$ versus $Q_{5}$ for all the crests. In figure 5 we show the actual shape of a non-symmetric wave.

The P-6 branch was computed using $n=256,512,768$ and 1024 to determine with 

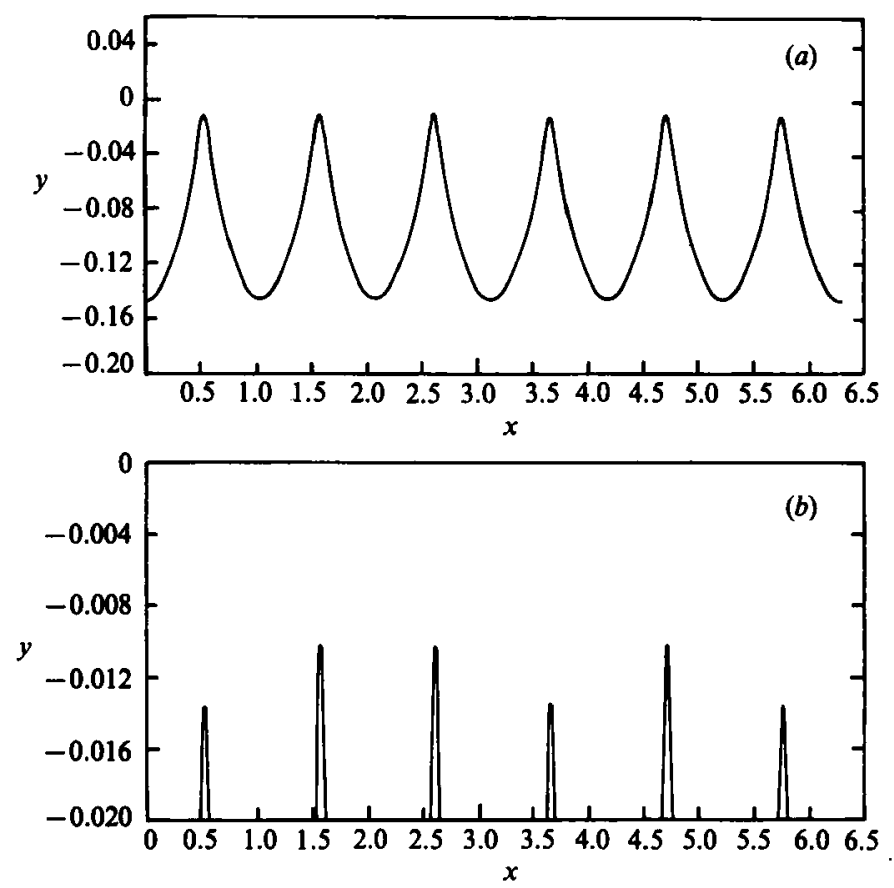

Figure 5. Non-symmetric gravity wave for $c=0.442497$. (a) Actual form of the wave.

(b) Detail of the relative amplitude of the crests.

\begin{tabular}{|c|c|c|c|c|c|c|c|c|}
\hline$c$ & $Q_{1}$ & $Q_{2}$ & $Q_{3}$ & $Q_{4}$ & $Q_{5}$ & $Q_{6}$ & $T$ & $V$ \\
\hline $0.442515 \dagger$ & 0.98794 & 0.98837 & 0.98794 & & & 0.98794 & 0.0064658 & 0.0059179 \\
\hline 0.442525 & 0.98777 & & & & & & & \\
\hline $2533 \ddagger$ & & & & & & & & \\
\hline 2539 & 0.9 & & 0.9 & & & & & 201 \\
\hline 2551 & 0.98 & & 0.9 & & & & & 0.0 \\
\hline 0.442571 & 0.98744 & 0.98854 & 0.98838 & & & & 0.0 & \\
\hline 0.442606 & 0.98721 & 0.98859 & 0.98855 & & 0.98859 & & & 0.0059258 \\
\hline 0.442640 & & & & & & & & \\
\hline & & & & & & & & \\
\hline & & & & & & & & \\
\hline 0449803 & 0.98562 & 0.98799 & 0.98948 & 0.98948 & 0.98799 & 0.98562 & 0.0064943 & 0.0059381 \\
\hline
\end{tabular}

TABLE 2. Values for some parameters of the wave along the P-6 branch

$\dagger$ Point $\boldsymbol{P}_{\mathbf{3}, \boldsymbol{\theta}}$. $\quad \ddagger$ Point $\boldsymbol{P}_{\mathbf{s b}}$.

high accuracy the position of the symmetry-breaking bifurcation. We found that with 1024 modes very good convergence is achieved. Along this branch the energy increases monotonically, as does the phase speed.

The non-symmetric branch P-6A was computed using only 256 and 512 modes in the non-symmetric code. This is equivalent from the computational point of view to using 512 and 1024 modes in the symmetric code. We can observe that the energy and the phase speed decay very rapidly along the non-symmetric family.

Properties of the waves along branches P-6 and P-6a are given in tables 2 and 3 . Table 4 shows the first 20 coefficients for the non-symmetric wave given in figure 5 . 


\begin{tabular}{|c|c|c|c|c|c|c|c|c|}
\hline$c$ & $Q_{1}$ & $Q_{2}$ & $Q_{3}$ & $Q_{4}$ & $Q_{5}$ & $Q_{6}$ & $T$ & $\boldsymbol{V}$ \\
\hline $442533+$ & 0.98769 & .98843 & 98816 & 98816 & 98843 & .98769 & 0.0064682 & 0.005919 \\
\hline 0.4 & .98748 & 0.988 & 0.98850 & 0.98780 & & 750 & .0064 & \\
\hline 2528 & 0.98724 & 0.988 & 0.98871 & 0.98756 & 0.98 & 0.98 & 0.0064662 & 0.0059178 \\
\hline & & & & & & & & 0.0059174 \\
\hline & & & & & & & & \\
\hline & & & & & & & & \\
\hline & & & & & & & & \\
\hline & & & & & & & & \\
\hline 475 & .98536 & 0.98 & 0.98986 & 0.98604 & 0.9 & 676 & 0.0064 & 0.0059006 \\
\hline 467 & 0.98515 & 0.98880 & 0.98995 & 0.98589 & 0.98 & 0.98561 & 0.0064 & 0.0058980 \\
\hline 0.442450 & 0.98473 & 0.98865 & 0.99012 & 0.98558 & 0.99007 & 0.98530 & 0.0064423 & 0.0058923 \\
\hline
\end{tabular}

TABLE 3. Values for some parameters of the wave along the P-6a branch

$\dagger$ Point $P_{\text {sb }}$

\begin{tabular}{rrr}
\hline & & \\
$j$ & $p_{j}\left(\times 10^{-3}\right)$ & $q_{j}\left(\times 10^{-3}\right)$ \\
0 & -216.795000 & 0 \\
1 & -0.092495 & 0.042022 \\
2 & 0.190116 & 0.099751 \\
3 & 0.003196 & 0.161178 \\
4 & -0.375653 & 0.194799 \\
5 & 0.444870 & 0.204610 \\
6 & -49.456600 & 0 \\
7 & -0.353888 & 0.158902 \\
8 & 0.197979 & 0.105166 \\
9 & 0.001469 & 0.025249 \\
10 & 0.102077 & -0.052641 \\
11 & -0.228032 & -0.106101 \\
12 & 16.506100 & -0.000028 \\
13 & 0.280234 & -0.100413 \\
14 & -0.186820 & -0.043752 \\
15 & -0.004249 & 0.021337 \\
16 & -0.423778 & 0.072821 \\
17 & 0.154760 & 0.000057 \\
18 & -8.425180 & 0.101246 \\
19 & -0.231066 & 0.089484 \\
20 & 0.164546 & \\
TABLE 4. Values of the first 20 coefficients for the non-symmetric wave shown in figure 5 \\
\end{tabular}

\section{Hamiltonian method}

\subsection{General formulation}

Consider two-dimensional irrotational periodic surface waves on water of infinite depth. Zakharov (1968) and Broer (1974) have proved that this system has the following Hamiltonian structure:

$$
\left.\begin{array}{c}
\frac{\partial \eta}{\partial t}=\frac{\delta \mathscr{H}}{\delta \psi}, \\
\frac{\partial \psi}{\partial t}=-\frac{\delta \mathscr{H}}{\delta \eta},
\end{array}\right\}
$$


where $\mathscr{H}$ is the total energy per period of the waves, and $\delta$ represents a functional derivative (see Goldstein 1980). The canonical variables $\eta(x, t)$ and $\psi(x, t)$ are the surface shape and the velocity potential evaluated at the surface $\psi(x, t)=$ $\Phi(x, \eta(x, t), t)$.

The lengthscale can always be chosen such that the period of the waves is $2 \pi$. With this choice $\eta(x, t)$ and $\psi(x, t)$ are $2 \pi$-periodic functions, and $\mathscr{H}$ is given by

$$
\mathscr{H}=\int_{0}^{2 \pi} H(\eta, \psi) \mathrm{d} x
$$

where $H$ is the energy density. In the case of gravity waves $H$ is given by

$$
H=\frac{1}{2} \int_{-\infty}^{\eta}(\nabla \Phi)^{2} \mathrm{~d} y+\frac{1}{2} g \eta^{2},
$$

where $g$ is the acceleration due to gravity, which is taken equal to 1 in the following.

As $\eta(x, t)$ and $\psi(x, t)$ are periodic functions of $x$, they can be expressed in the following form:

$$
\left.\begin{array}{c}
\eta(x, t)=\sum_{-\infty}^{\infty} \eta_{k}(t) \mathrm{e}^{\mathrm{i} k x}, \\
\psi(x, t)=\sum_{-\infty}^{\infty} \psi_{k}(t) \mathrm{e}^{i k x},
\end{array}\right\}
$$

where $\eta_{k}=\eta_{-k}^{*}$ and $\psi_{k}=\psi_{-k}^{*}$ because $\eta$ and $\psi$ are real. The transformation defined by (19) can be considered as a canonical transformation from a continuous system with canonical variables $\eta(x, t)$ and $\psi(x, t)$ into an infinite dimensional discrete system with the infinite sets $\left\{\eta_{k}\right\}$ and $\left\{\psi_{k}\right\}$ as canonical variables (Goldstein 1980).

To write the system in a more compact form, Zakharov (1968) introduced the following transformation:

$$
\left.\begin{array}{c}
\eta_{k}(t)=\frac{|k|^{\frac{1}{2}}}{2 \pi^{\frac{1}{2}} \omega^{\frac{1}{2}}(k)}\left[a_{k}(t)+a_{-k}^{*}(t)\right], \\
\psi_{k}(t)=-\frac{\mathrm{i} \omega^{\frac{1}{2}}(k)}{2 \pi^{-\frac{1}{2}|k|^{\frac{1}{2}}}\left[a_{k}(t)-a_{-k}^{*}(t)\right],}
\end{array}\right\}
$$

where $\omega(k)$ is the linear dispersion relation for deep-water waves, which is given by

$$
\omega(k)=|k|^{\frac{1}{2}} .
$$

When the horizontal level of the frame of reference is chosen at the mean horizontal level of the surface, $a_{0}=0$.

Using transformations (19) and (20), the Hamiltonian system (16) becomes the single equation

where now

$$
\frac{\partial a_{k}}{\partial t}=-\mathrm{i} \frac{\partial \mathscr{H}}{\partial a_{k}^{*}},
$$

and $a=\left\{a_{k}\right\}$. Notice that the functional derivative has become a partial derivative because we now have a discrete system.

As we are interested in the study of permanent-form travelling waves, it is convenient to write (22) in a coordinate system moving with the phase speed $c$ :

$$
\frac{\partial a_{k}}{\partial t}=-\mathrm{i} \frac{\partial \mathscr{H}}{\partial a_{k}^{*}}+\mathrm{i} c k a_{k}
$$


Travelling waves of permanent form are equilibrium solutions (fixed points) of this system. For symmetric wave solutions, the origin of the reference frame can always be chosen such that $a_{k}=a_{k}^{*}$. An important property of $\mathscr{H}$ is that $\mathscr{H}$ is invariant under the change $a_{k} \rightarrow a_{k}^{*}$.

Instead of considering only (24), it is convenient to consider (24) together with its complex conjugate and the coefficients $\left\{a_{k}\right\}$ and $\left\{a_{k}^{*}\right\}$ as different independent variables. With these considerations the system is analytic, and the stability analysis becomes clearer.

The stability of these equilibrium solutions of (24) under superharmonic perturbations $\left(\propto \mathrm{e}^{\mathrm{i} \sigma t}\right.$ ) is determined by the eigenvalues of the matrix (Saffman 1985; Zufiria \& Saffman 1986)

where

$$
S\left(a, a^{*}\right)=\left(\begin{array}{cc}
M & N \\
-N^{*} & -M
\end{array}\right)
$$

When all the eigenvalues are real the system is said to be stable. Stability is lost when some of the eigenvalues leave the real axis. This can occur only by collision of two eigenvalues of different signature (MacKay 1986; MacKay \& Saffman 1986).

Some important properties of the matrix $S$ called 'Hamiltonian' (Arnol'd 1978) are that if $\sigma$ is an eigenvalue of $S$, so is $-\sigma$, and so are the complex conjugates $\sigma^{*}$ and $-\sigma^{*}$. The matrix $S$ always has eigenvalue that is zero due to the Galilean invariance. Because the system is even-dimensional $\sigma=0$ has to be at least a double eigenvalue. Saffman (1985) proved that at points where the wave energy is an extremum $\sigma=0$ is an eigenvalue of algebraic multiplicity four and geometric multiplicity one. This analytical result was checked by our computations, as we shall see below.

As happened in the Stokes formulation, it is important to notice that if a set of coefficients $\left\{a_{k}\right\}$ represents an equilibrium solution of (24) the set $\left\{a_{k}^{N}\right\}$ where

$$
\left.\begin{array}{l}
a_{k N}^{N}=\left(\frac{1}{N}\right)^{\frac{5}{4}} a_{k}, \\
a_{k}^{N}=0 \text { otherwise, }
\end{array}\right\}
$$

is also an equilibrium solution of (24), and represents a class- $N$ wave.

\subsection{Weakly nonlinear form}

Before going into the details of the numerical algorithm, it is worthwhile to work out some linear results that will help us to generate starting points and check the computations.

For linear waves the Hamiltonian $\mathscr{H}$ is given by

$$
\mathscr{H}=\sum_{-\infty}^{\infty} \omega(k) a_{k} a_{k}^{*}
$$

Using (24) the equations for travelling waves are

$$
\left(|k|^{\frac{1}{2}}-c k\right) a_{k}=0 \quad(-\infty<k<\infty) .
$$

We have an eigenvalue problem. For $c \neq 1 /|k|^{\frac{1}{2}}$ there is a unique solution $a_{k}=0$ for all $k$, that corresponds to the flat surface. For $c=1 /|k|^{\frac{1}{2}}$ we have a bifurcation point for a regular class- $k$ wave $\left(a_{j}=0\right.$ for $j \neq k$ and $a_{k}$ arbitrary). 
The eigenvalues for the superharmonic stability of the solutions at the bifurcation points are

$$
\sigma_{j}^{k}= \pm\left[|j|^{\frac{1}{2}}+\frac{j}{|k|^{\frac{1}{2}}}\right] .
$$

These values will serve as a first check of the stability code. Each eigenvalue is named by giving the two numbers $j / k$. The eigenvector associated to the eigenvalue $j / k$ is an oscillation with $|j|$ crests on a class- $k$ wave. Negative values of $j$ correspond to perturbations travelling in the same sense as the unperturbed wave. Positive values of $j$ correspond to perturbations travelling in the opposite sense to the unperturbed wave.

As we are interested in the branch corresponding to class 6 waves, in order to generate a starting point for the computations we calculated the expansion of the energy for small amplitude to second-order, obtaining

$$
\mathscr{H} \approx \sqrt{ } 6\left[a_{6} a_{6}^{*}+\frac{9}{2 \pi} \sqrt{ } 6 a_{6}^{2} a_{6}^{* 2}\right] .
$$

With this Hamiltonian, we find that the beginning of the class 6 branch is given by

$$
a_{6} a_{6}^{*} \approx \frac{\sqrt{ } 6 \pi}{162}[\sqrt{ } 6 c-1]
$$

\subsection{Numerical algorithm}

We are interested in computing travelling waves of permanent form. In a frame moving with the wave, these waves are solutions of the following steady-state

$$
\left.\begin{array}{ll}
\frac{\partial \mathscr{H}}{\partial a_{k}^{*}}-c k a_{k}=0 & \\
\frac{\partial \mathscr{H}}{\partial a_{k}}-c k a_{k}^{*}=0 & (-\infty<k<\infty),
\end{array}\right\}
$$

where the equations are complex.

We now truncate the system (32) by taking $a_{k}=0$ for $|k|>n$. Thus we shall consider only $2 n$ complex coefficients to describe the wave. Then we have to solve an algebraic system of $4 n$ equations in $4 n$ unknowns.

To make this formulation useful, we first need to compute the gradient of the energy $\mathscr{H}$ in terms of the set of coefficients $\left\{a_{k}\right\}$. In other words, we have to evaluate (18) and its derivatives in terms of the coefficients. The part corresponding to the potential energy is straightforward. The most difficult part is to compute the kinetic energy. Notice that as we need to compute the gradient of the energy we have to compute the energy of a general water wave which in general will not correspond to a permanent-form travelling wave. Hence simple expressions for the energy similar to the ones shown in (11) and (12), which are valid only for steady travelling waves, cannot be used for the present purpose. Here we describe a way of doing this computation.

Knowing the set of coefficients $\left\{a_{k}\right\}$, we can directly compute the sets $\left\{\eta_{k}\right\}$ and $\left\{\psi_{k}\right\}$ using (20). To evaluate the kinetic energy we need to know the velocity potential $\Phi$ in the fluid region. Using Fourier analysis and assuming that $\Phi$ is analytic below the line of mean horizontal height, we can write

$$
\Phi(x, y)=\sum_{k=-\infty}^{\infty} \Phi_{k} \mathrm{e}^{|k| y} \mathrm{e}^{\mathrm{i} k x}
$$


where the set $\left\{\Phi_{k}\right\}$ is the coefficients of the Fourier expansion of the velocity potential on the line $y=0$.

Introducing (33) into (18) and performing the integral, we obtain the following expression for the total energy:

$$
\mathscr{H}=4 \pi \sum_{k=1}^{\infty} \sum_{j=1}^{\infty} \frac{k j}{k+j} \Phi_{k} \Phi_{-j} C_{j-k, k+j}+2 \pi \sum_{k=1}^{\infty} \eta_{k} \eta_{-k}
$$

where the coefficients $C_{k, m}$ are defined by

$$
C_{k, m}=\frac{1}{2 \pi} \int_{0}^{2 \pi} \mathrm{e}^{-\mathrm{i} k x} \mathrm{e}^{|m| \eta(x)} \mathrm{d} x .
$$

These coefficients depend only on the set $\left\{\eta_{k}\right\}$, and can be computed in the following way. Using an inverse fast Fourier transform (FFT) we can compute $\eta(x)$ evaluated on equidistant nodes from the set $\left\{\eta_{k}\right\}$. For every value of $m$ we take the exponential, and via a forward FFT we obtain the coefficients $C_{k, m}$ directly. An alternative way of computing these coefficients consists of expanding the exponential function in Taylor series, and after doing the algebra among the different modes we can obtain $C_{k, m}$. For small-amplitude waves the last procedure is faster because we only need to take a few terms in the Taylor expansion of $\exp (|m| \eta(x))$. For the range of amplitudes in which we are interested, we found that the expansion has to be carried out up to order 30 to get convergence. Hence for our purposes the algorithm based on the FFT was found to be faster.

To compute the coefficients $\Phi_{k}$ we write (33) on the surface of the wave in the following form:

$$
\sum_{k=-n}^{n} \psi_{k} \mathrm{e}^{\mathrm{i} k x}=\sum_{j=-\infty}^{\infty} \Phi_{j} \mathrm{e}^{|j| \eta(x)} \mathrm{e}^{\mathrm{i} j x}
$$

Introducing the coefficients $C_{k, m}$ and equating the same harmonics, (36) can be rewritten as follows:

where $\psi_{j}=0$ for $j>n$.

$$
\psi_{j}=\sum_{k=-\infty}^{\infty} \Phi_{k} C_{j-k, k} \quad(-\infty<j<\infty),
$$

It is important to notice that the series in (36) and (37) will be uniformly convergent if and only if the analytic continuation of $\Phi(x, y)$ above the surface does not have any singularity below the highest crest of the wave. For the waves that we shall consider this was found to be true.

We now have to truncate the set $\left\{\Phi_{k}\right\}$, and take $\Phi_{k}=0$ for $|k|>n_{\mathrm{a}}$. Thus we have two parameters in the algorithm, $n$ and $n_{\mathrm{a}}$. How the algorithm converges as $n_{\mathrm{a}}$ is increased serves as a measure of the validity of the statement of the previous paragraph.

To compute the set $\left\{\Phi_{k}\right\}$ we need to solve the linear system of equations defined by (37). 'This part of the algorithm has a complexity of $n_{\mathrm{a}}^{3}$ operations and is the time consuming part of the method.

Until now we have only computed the energy $\mathscr{H}$, but for (32) we actually need to compute the gradient of the energy. This can be done without increasing the complexity of the algorithm as follows.

Taking the derivative of (34) with respect to $a_{l}^{*}$ we have

$$
\frac{\partial \mathscr{H}}{\partial a_{l}^{*}}=4 \pi \sum_{k=1}^{n_{\mathrm{a}}} \sum_{j=1}^{n_{\mathrm{a}}} \frac{k j}{k+j}\left\{\left[\frac{\partial \Phi_{k}}{\partial a_{l}^{*}} \Phi_{-j}+\Phi_{k} \frac{\partial \Phi_{-j}}{\partial a_{l}^{*}}\right] C_{j-k, k+j}+\Phi_{k} \Phi_{-j} \frac{\partial C_{j-k, k+j}}{\partial a_{l}^{*}}\right\}+\frac{1}{2} \pi^{\frac{1}{2}}|l|^{1} \eta_{l} .
$$


The derivative of the coefficients $C_{k, m}$ can be directly obtained by differentiation of (35), giving

$$
\frac{\partial C_{k, m}}{\partial a_{l}^{*}}=\frac{\partial C_{k, m}}{\partial \eta_{-l}} \frac{|k|^{\frac{1}{4}}}{2 \pi^{\frac{1}{2}}}=\frac{|k|^{\frac{1}{3}}}{2 \pi^{\frac{1}{2}}} C_{k+l, m} .
$$

To obtain the derivatives of the coefficients $\Phi_{k}$ we take the derivative of (37) with respect to $a_{l}^{*}$. We have

$$
\frac{\partial \psi_{j}}{\partial a_{l}^{*}}-\sum_{k=-n_{\mathrm{a}}}^{n_{\mathrm{a}}} \phi_{k} \frac{\partial C_{j-k, k}}{\partial a_{l}^{*}}=\sum_{k=-n_{\mathrm{a}}}^{n_{\mathrm{a}}} C_{j-k, k} \frac{\partial \Phi_{k}}{\partial a_{l}^{*}}
$$

Therefore to compute the derivatives of $\Phi_{k}$ we have to solve $2 n$ linear systems of equations. But notice that we only need to make one back substitution, because the matrix of the system is the same as the one that we had before in order to compute the set $\left\{\Phi_{k}\right\}$. Thus to compute the set of derivatives $\partial \Phi_{j} / \partial a_{l}^{*}$ we need $2 n$ back substitutions. The total complexity of this part is $n n_{\mathrm{a}}^{2}$.

Notice that using this algorithm, the expansion that we are actually obtaining for the function $\mathscr{H}\left(a, a^{*}\right)$ is not a Taylor polynomial expansion, as happens in the weakly nonlinear expansion given by Zakharov (1968). Due to the properties of the linear system that we are solving, the expansion has the form of the ratio of two polynomials. The structure is similar to the one that is obtained when using Padé approximants to represent a function.

Once we can compute the gradient of the energy in terms of the coefficients $a_{k}$, we can solve (32) by using arc-length continuation (Keller 1977) in the parameter c. As happened in the Stokes expansion method, the system defined by (32) is degenerate owing to the Galilean invariance. As can be very easily checked, if a set of coefficients $\left\{a_{k}\right\}$ represents a solution of (32) the set $\left\{a_{k} \mathrm{e}^{\mathrm{i} k \varepsilon}\right\}$ is also a solution of (32) for all $\epsilon$. Therefore, as we have already seen, the Jacobian of (32) (matrix $S$ ) is singular.

To be able to do the continuation with this degeneracy, we modified the continuation algorithm by imposing the condition that the increments in the solution as the parameter is changed be normal to the null vector of the Jacobian. The null vector of the Jacobian is given by the set $\left\{\mathrm{i} k a_{k}\right\}$. This is equivalent to performing the continuation in a subspace that is the projection of the whole space on to the direction normal to the null vector. Physically this is equivalent to keeping the origin fixed.

Equation (32) represents a set of $4 n$ complex equations for the coefficients $a_{k}$ and $a_{k}^{*}$. Using linear combinations among the equations and taking $a_{k}=p_{k}+\mathrm{i} q_{k}$, the system can be reduced to $4 n$ real equations for the coefficients $p_{k}$ and $q_{k}$.

For the continuation process the Jacobian was computed numerically using increments. Even though we used double precision in our calculations, this was the main source of inaccuracy in the computations of the eigenvalues for the stability.

The whole algorithm can be simplified when considering symmetric waves. For symmetrical solutions the coefficients can be taken to be real, with $a_{k}=a_{k}^{*}$. Then the original system (32) can be reduced to only the first set of $2 n$ real equations. The Jacobian of this real system is just the matrix $M-N$, where $M$ and $N$ have already been defined in (25).

\subsection{Numerical results}

In order to check our code we first computed the regular branch using a class 1 wave. Taking $n=12, n_{\mathrm{a}}$ was increased until convergence was obtained for the solution in the range from zero amplitude up to the amplitude at which the energy 


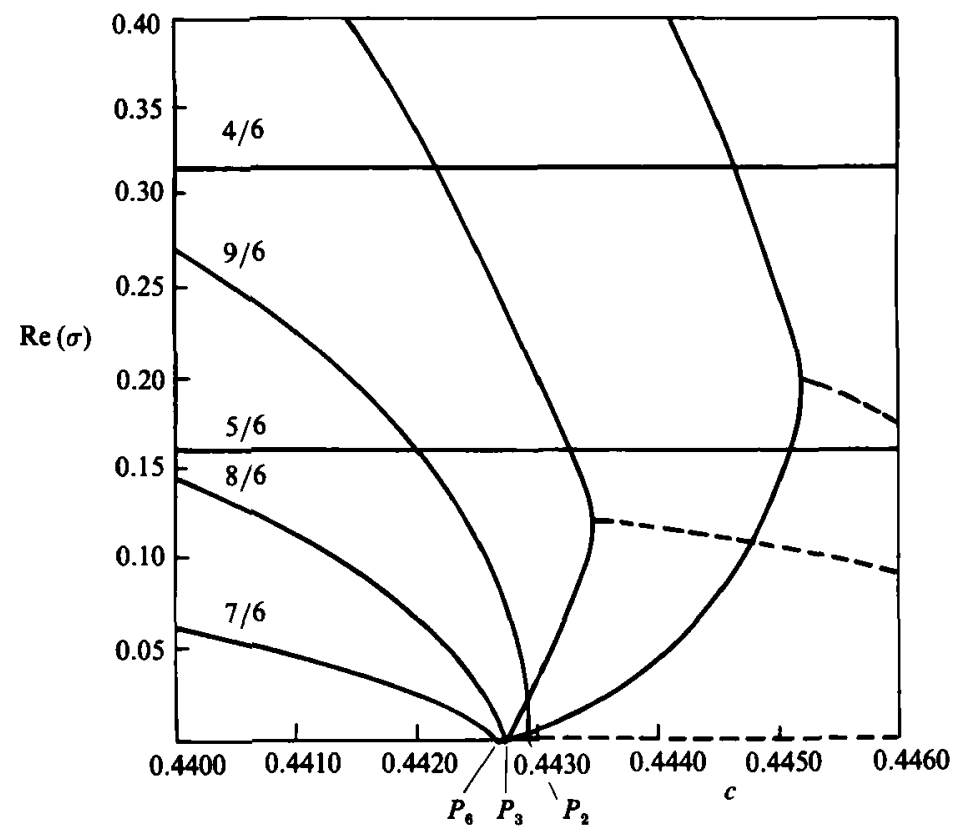

FIGURE 6. Eigenvalues of the superharmonic stability of the class 6 regular family showing the bifurcation points. Dashed lines denote $\sigma$-complex.

of the wave reaches the first maximum. We found that $n_{\mathrm{a}}=2 n=24$ is sufficient to get convergence in this range.

All the eigenvalues of the matrix $\boldsymbol{S}$ were computed along this branch. We found that an exchange of stability occurs at the point of maximum energy. This result was first found by Tanaka (1983), who studied the superharmonic stability of regular Stokes waves using a numerical method based on conformal-mapping techniques. Later Saffman (1985) proved the result analytically, using the Hamiltonian formulation of the water-wave problem. He found that it is a property of the Hamiltonian structure of the problem. Because the code keeps the Hamiltonian structure, the coincidence of the critical amplitude for the superharmonic stability and the amplitude for the maximum energy is a property of the structure of the code, and is independent of the number of modes, even though the location of the actual point is inaccurate unless the number of modes is sufficiently large. On the other hand, other methods like the Stokes expansion used by Longuet-Higgins $(1978 b)$ and the methods used by Tanaka $(1983,1985)$ are inaccurate in both respects unless the number of modes is sufficiently large, i.e. when a small number of modes are used in the computations both points do not coincide and their location is not accurate. We also found that results for the eigenvalues of the first few modes are in very good agreement with the computations of Longuet-Higgins $(1978 b)$ in the range of steepness within which his results are valid.

With $n=12$ we found good numerical convergence in the region of amplitudes where bifurcations to other families are expected to happen. Actually in this region we found that $n=n_{\mathrm{a}}$ gives convergence for a given $n$, and it is not necessary to go up to $n_{\mathrm{a}}=2 n$.

In the region of strong nonlinearity where the extrema of the energy and phase speed appear, with $n=12$ and $n_{\mathrm{a}}=24$ we were able to reproduce the first maximum 


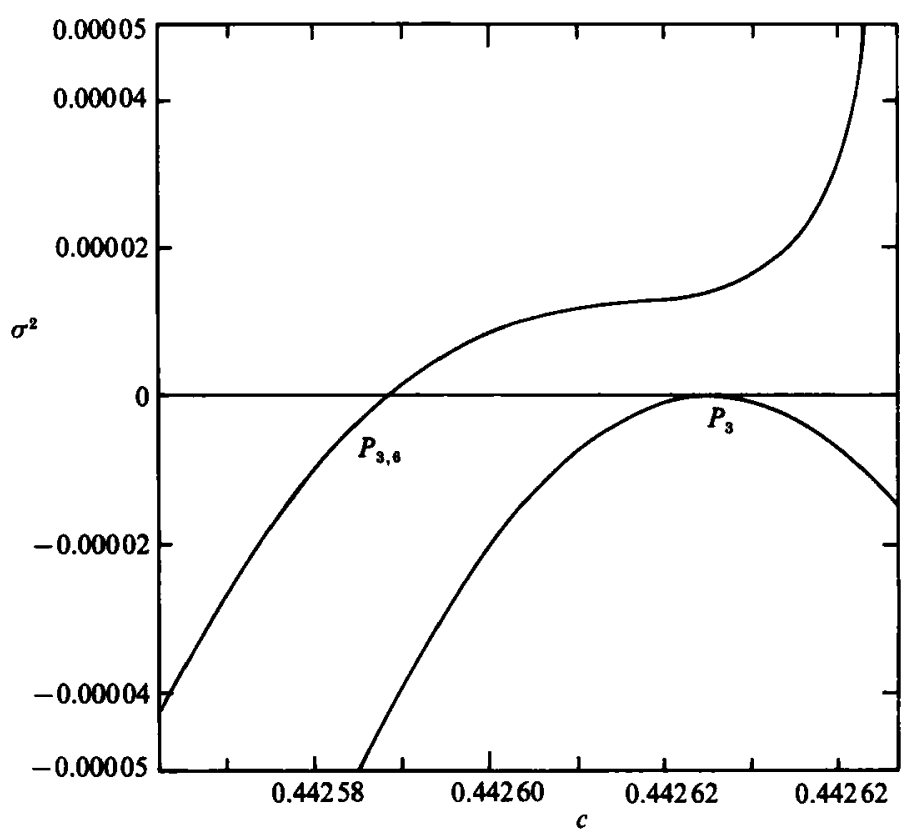

Figure 7. Behaviour of the eigenvalues along branch P-3, associated with the bifurcation at $P_{3}$ and $P_{3,6}$.

of the energy and the phase speed. Numerical values were within $7 \%$ of the real ones for the phase speed and $2.5 \%$ for the energy and the amplitude.

Once the code was checked, we tried to reproduce the results for symmetry breaking that we obtained by the Stokes expansion. Using (31) we generated a starting point for the regular class 6 family. We increased the amplitude up to the point where bifurcations appear. As the eigenvalues were computed the bifurcations were checked by looking at where eigenvalues become zero. Eigenvalues along this branch are plotted in figure 6.

Because of the complexity of the algorithm, computations could be carried out only for up to $n=72$. This is equivalent to $n=12$ in a class 1 wave. To switch branches we used the same procedure as in the previous method, and again we found that the method converges to the new branches in three or four iterations.

Figure 6 shows eigenvalues for disturbances to class 6 waves. We can see that there are three bifurcation points. These bifurcations are the same as the ones obtained by the Stokes formulation. Notice that only the period-doubling bifurcation leads to a change of stability in the regular family. At the other two points eigenvalues pass through zero, but they do not give a change of stability. From the behaviour of the eigenvalues it is probable that more bifurcations exist for higher amplitudes. However, in that region we do not get convergence with the number of modes that we are using. These higher-amplitude bifurcations were computed by Vanden-Broeck (1983) using conformal-mapping techniques for finite-depth water waves. Values of the phase speed and total energy for the period-tripling bifurcation $\left(P_{3}\right)$ are given in table 1.

At the period-tripling bifurcation, we switched to the new branch (P-3). This branch was computed in the direction of decreasing phase speed. We found that there 


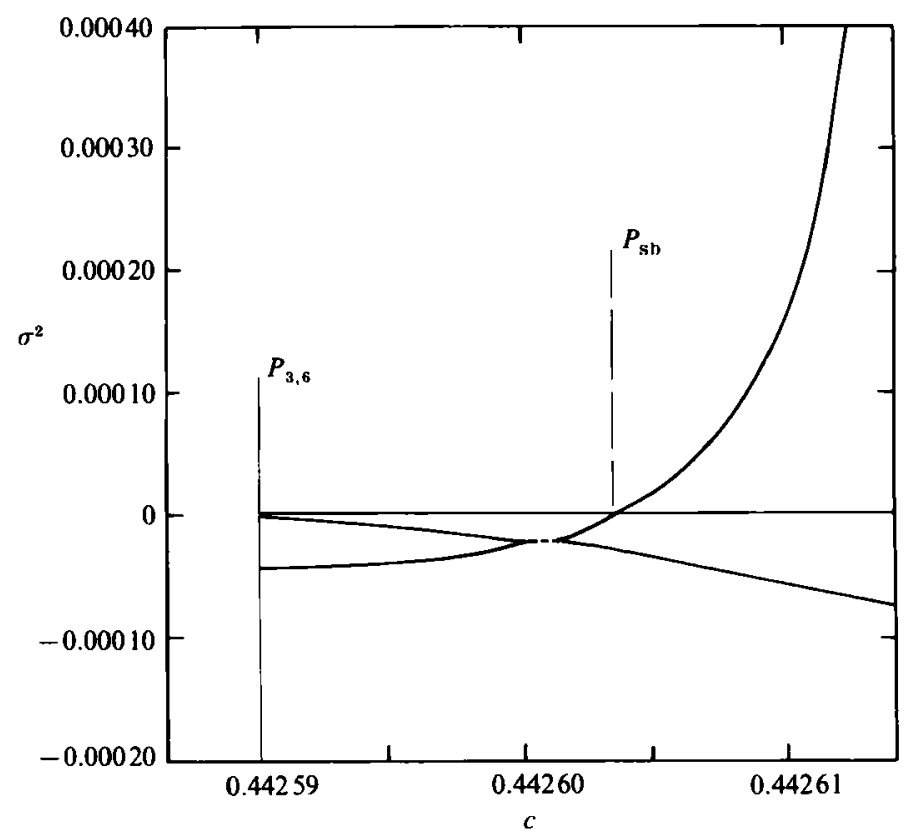

Figurs 8. Behaviour of the eigenvalues along branch P-6, associated with the bifurcations at $P_{3,6}$ and $P_{\text {sb }}$.

\begin{tabular}{lcccc}
\hline & $n$ & $c$ & $E=T+V$ & $Q_{2}$ \\
Stokes expansion & 256 & 0.442988 & 0.0125027 & 0.988756 \\
& 512 & 0.442515 & 0.0123836 & 0.988367 \\
& 768 & 0.442510 & 0.0123825 & 0.988372 \\
Hamiltonian method & 1024 & 0.442510 & 0.0123825 & 0.988373 \\
& 60 & 0.442918 & 0.0124842 & \\
& 72 & 0.442590 & 0.0124018 &
\end{tabular}

TABLE 5. Values obtained for some parameters of the wave at the bifurcation point $P_{3,6}$

is always at least one unstable eigenvalue on this branch. In figure 7 we show the two smallest eigenvalues, besides the one due to the shift $(\sigma=0)$. The eigenvalue that leads to the $P_{3}$ bifurcation is the one that is always unstable. This was expected, because on the P-1 branch there is no change of stability at the $P_{3}$ bifurcation point, as can be seen in figure 6 . We can see that the other eigenvalue also goes through zero, giving a new bifurcation. This bifurcation is a period-doubling bifurcation. Actually, this is the point $P_{3,6}$ in figure 2 .

At the point $P_{3,6}$ we switched to the new branch (P-6). The eigenvalues along the new branch are given in figure 8 . Again we plot the two smallest eigenvalues, finding that another bifurcation appears on this branch. This bifurcation corresponds to the symmetry breaking. In figure 8 we can also see that the eigenvalue that gives the $P_{3,6}$ bifurcation is unstable along the $\mathrm{P}-6$ branch. Notice that, as $P_{3,6}$ is period-doubling bifurcation, the new branch P-6 starts at the bifurcation point and does not exist for values of the phase speed below the value corresponding to the bifurcation point. 


$\begin{array}{lrccc} & n & c & E=T+V & Q_{2} \\ \text { Stokes expansion } & 256 & 0.443014 & 0.0125082 & 0.988823 \\ & 512 & 0.442533 & 0.0123874 & 0.988429 \\ & 768 & 0.442528 & 0.0123863 & 0.988434 \\ \text { Hamiltonian method } & 1024 & 0.442529 & 0.0123866 & 0.988440 \\ & 60 & 0.442938 & 0.0124886 & \\ & 72 & 0.442606 & 0.0124052 & \end{array}$

TABLE 6. Values obtained for some parameters of the wave at the bifurcation point $P_{\mathrm{sb}}$

Values of the phase speed and energy at the point $P_{3,6}$ and $P_{\mathrm{sb}}$ are given in tables 5 and 6 respectively.

Therefore we find the same bifurcation structure using this completely different numerical approach to the problem. This is strong evidence for the validity of our results.

\section{Conclusions}

We have shown that non-symmetric gravity waves exist on deep water. They appear from a spontaneous symmetry-breaking bifurcation of symmetric waves. To find one of these bifurcations we go from the regular family of waves to an irregular family of class 3, which was found by Chen \& Saffman (1980). In this class 3 family there is a period-doubling bifurcation to a family of waves that have six crests per basic wavelength. This class 6 wave can bifurcate to a non-symmetric family also with six crests per period.

Hence we find that results found by Zufiria (1987) using a weakly nonlinear Hamiltonian model for finite-depth water waves can be completely generalized to the full system and to the deep-water case. Zufiria's (1987) results follow from properties of two-degrees-of-freedom Hamiltonian systems. This means that in this case results for a finite-dimensional Hamiltonian system appear to apply for a continuous system. This raises again the open question of whether or not travelling water waves of permanent form are actually a Hamiltonian system of two degrees of freedom.

In this way Hamiltonian methods have served to answer one more question in the problem of water waves.

In the present paper we have computed only one family of non-symmetric waves. This does not mean that this family is unique. For area-preserving maps many more symmetry-breaking bifurcations have been found, but this one is the easiest to compute in the case of water waves. The other bifurcations appear with considering waves of higher class. Class 6 is the minimum class to have symmetry breaking.

To confirm all these results we have performed computations using two different methods. The first is based on the Stokes expansion and is shown to be very easy to implement and fast. Therefore many modes can be used in the computations, and the method gives the most accurate results. But we have found that the structure of the equations is poor in the sense that the number of modes needed to reproduce the physical behaviour and to find convergence is large.

The new numerical method based on the Hamiltonian formulation of the waterwaves problem has been found to be much more expensive from the computational 
point of view because of the complexity of the algorithm to compute the energy. We have been able to run only up to 72 modes, but the bifurcation structure was reproduced using as few as 12 modes.

Also the algorithm gives the stability of the wave solutions without much additional work. The method can be very easily generalized to other cases. For example, to consider gravity-capillary waves we only need to add to the total energy the energy due to the surface tension without changing the main part of the code. Also, the same code can be used for studying unsteady problems in water waves, and keeping the Hamiltonian structure of the problem. For example, in initial-value problems properties that have to be conserved such as energy are directly checked.

More work should be done to try to speed up the way of computing the kinetic energy, so that the computations can be made more efficient. Also generalization to three-dimensional waves would be straightforward.

I am indebted to Professor P. G. Saffman for his encouragement and valuable advice during the course of the research. This work was supported by the Office of Naval Research (N00014-79-C-0412, NR062-639) and by the National Science Foundation (OCE-8415988). I also wish to acknowledge receipt of a Fulbright award.

\section{REFERENCES}

Amick, L. J., Fraenkel, L. E. \& Toland, J. F. 1982 On the Stokes conjecture for the wave of extreme form. Acta Math., Stockh. 148, 193-214.

ARnol'D, V. I. 1978 Mathematical Methods of Classical Mechanics. Springer.

ARnol'D, V. I. \& Avez, A. 1968 Ergodic Problems of Classical Mechanics. Benjamin.

Brokr, L. J. F. 1974 On the Hamiltonian theory of surface waves. Appl. Sci. Res. 29, 430-446.

BoussinesQ, J. 1871 Théorie l'intumescence liquide appelée onde solitaire ou de translation se propageant dans un canal rectangulaire. C.R. Acad. Sci. Paris 72, 755-759.

Chen, B. \& Saffman, P. G. 1980 Numerical evidence for the existence of new types of gravity waves of permanent form on deep water. Stud. App. Maths 62, 1-21.

Goldstein, H. 1980 Classical Mechanics, 2nd edn. Addison Wesley.

Green, J. M., Mackay, R. S., Vivaldi, F. \& Feigenbaum, M. J. 1981 Universal behaviour in families of area-preserving maps. Physics $3 \mathrm{D}, \mathbf{4 6 8 - 4 8 6 .}$

KELLER, H. B. 1977 Numerical solution of bifurcation and nonlinear eigenvalue problems. In Applications of Bifurcation Theory (ed. P. W. Rabinowitz), pp. 359-384. Academic.

Korteweg, P. J. \& DE VRIES, G. 1895 On the change of form of long waves advancing in a rectangular channel, and on a new type of long stationary waves. Phil. Mag. 39 (5), 422-443.

Longuet-Higgins, M. S. $1978 a$ Some new relations between Stokes's waves coefficients in theory of gravity waves. J. Inst. Maths Applics 22, 261-273.

Longuet-Higans, M. S. $1978 b$ The instabilities of gravity waves of finite amplitude in deep water. I. Superharmonics. Proc. R. Soc. Lond. A 360, 471-488.

Longuet-Higarns, M.S. 1984 New integral relations for gravity waves of finite amplitude. J. Fluid Mech. 149, 205-215.

Longuet-Higains, M. S. 1985 Bifurcation in gravity waves. J. Fluid Mech. 151, 457-475.

MaCKAY, R. S. 1986 Stability of equilibria of Hamiltonian systems. In Nonlinear Phenomena and Chaos (ed. S. Sarkar). Adam Hilger.

MacKay, R. S. \& Saffman, P. G. 1986 Stability of water waves. Proc. R. Soc. Lond. 406, 115-125.

Rayleigh, Lord 1876 On waves. Phil. Mag. 1 (5), 257-279.

SAFFMAN, P. G. 1980 Long wavelength bifurcation of gravity waves on deep water. J. Fluid Mech. $101,567-581$. 
Saffman, P. G. 1985 The superharmonic instability of finite-amplitude water waves. $J$. Fluid Mech. 159, 169-174.

SATtinger, D. H. 1980 Bifurcation and symmetry breaking in applied mathematics. Bull. Am. Math. Soc. 3, 779-819.

SAtTinger, D. H. 1983 Branching in the presence of symmetry. CBMS-NSF Reg. Conf. in Appl. Math. SIAM, Philadelphia.

Schwartz, L. W. 1974 Computer extension and analytic continuation of Stokes' expansion for gravity waves. J. Fluid Mech. 63, 553-578.

Simmen, J. A. \& Saffman, P. G. 1985 Steady deep-water waves on a linear shear current. Stud. Appl. Maths 73, 35-57.

Stokes, G. G. 1849 On the theory of oscillatory waves. Trans. Camb. Phil. Soc. 8, 441-455.

TAnAKa, M. 1983 The stability of steep gravity waves. J. Phys. Soc. Japan 52, 3047-3055.

Tanaka, M. 1985 The stability of steep gravity waves. Part 2. J. Fluid Mech. 156, 281-289.

VANDEN-Broeck, J. M. 1983 Some new gravity waves in water of finite depth. Phys. Fluids 26, 2385-2387.

ZakHAROV, V. E. 1968 Stability of periodic waves of finite amplitude on the surface of deep fluid. J. Appl. Mech. Tech. Phys. 2, 190-194.

ZUFIRIA, J. A. 1987 Weakly nonlinear nonsymmetric gravity waves on water of finite depth. J. Fluid Mech.

Zufiria, J. A. \& Saffman, P. G. 1986 The superharmonic instability of finite amplitude surface waves on water of finite depth. Stud. Appl. Maths 74, 259-266. 